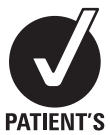

CHOICE

\title{
The natural history of secondary progressive multiple sclerosis
}

\author{
Marcus Koch, ${ }^{1,2}$ Elaine Kingwell, ${ }^{1}$ Peter Rieckmann, ${ }^{1}$ Helen Tremlett, ${ }^{1}$ UBC MS Clinic \\ Neurologists
}

\section{See Editorial Commentary, p 944}

${ }^{1}$ Faculty of Medicine, Division of Neurology, University of British Columbia, Vancouver, Canada ${ }^{2}$ Department of Neurology, University Medical Centre Groningen, University of Groningen, Groningen, The Netherlands

\section{Correspondence to}

Dr M Koch, Department of Neurology, University Medical Centre Groningen, Hanzeplein 1 , 9713 GZ Groningen, The Netherlands;

m.w.koch@neuro.umcg.nl

UBC MS Neurologists: D Adams, D Craig, L Daly,

$\checkmark$ Devonshire, S Hashimoto,

0 Hrebicek, J Hooge, B Jones,

L Kastrukoff, S Meckling,

J Oger, D Parton, D Paty,

P Smyth, W Shtybel,

T Traboulsee.

Received 3 February 2010 Revised 5 April 2010 Accepted 6 April 2010 Published Online First 16 July 2010

\section{ABSTRACT \\ Background The onset of secondary progression is} a pivotal event in the course of relapsing-remitting (RR) multiple sclerosis (MS). Patients with secondary progressive MS (SPMS) experience continuous worsening of symptoms independent of the occurrence of relapses. Possible risk factors associated with the onset of SPMS remain under investigated in natural history studies of MS disease course.

Methods We used Kaplan-Meier survival analyses and Cox regression models to investigate the influence of gender, onset age and onset symptoms on time to and age at SPMS in British Columbia (BC) MS patients with a RR disease onset who were not exposed to immunomodulatory drugs.

Results Of 5778 patients in the BCMS database with definite MS, 5207 (90\%) had an RR onset. Median time to SPMS was 21.4 years (95\% Cl 20.6 to 22.2 ), reached at a median age of 53.7 years $(95 \% \mathrm{Cl} 53.1$ to 54.3$)$. Male gender and motor onset symptoms were associated with a shorter time to and a younger age at SPMS. A younger age at disease onset was associated with a longer time to SPMS but also with a younger age at secondary progression. Other onset symptoms were not associated with time to, or age at, SPMS.

Conclusions We identified three factors influencing the onset of SPMS in untreated patients with RRMS: motor onset symptoms and male gender were associated with both a shorter time to and a younger age at SPMS. A younger age at disease onset should not be viewed as indicating a better prognosis.

\section{INTRODUCTION}

Natural history studies of multiple sclerosis (MS) often focus on the time from disease onset until patients reach certain landmark disability scores (such as Expanded Disability Status Scale (EDSS) 6.0, when a cane is required for walking, or EDSS 8.0, when a patient is mostly restricted to a wheelchair). Besides the accumulation of disability, patients with relapsing-remitting $\mathrm{MS}$ are also at risk of experiencing a critical change in their disease course-the development of secondary progression.

A secondary progressive disease course has developed when a patient's clinical condition starts to deteriorate steadily and independently of relapses. Patients with secondary progressive MS (SPMS) have an unfavourable prognosis and can expect a slow and unremitting worsening of their ability to function. ${ }^{2}$ The onset of secondary progression is therefore another important outcome to examine in natural history studies.
The onset of secondary progression has been addressed by several small studies that may have had limited power and have often focused on patients who had already reached SPMS. ${ }^{3-5}$ Tremlett et al have previously investigated a subset of the British Columbia MS (BCMS) cohort $^{6}$ but when examining predictors of progression were unable to fully adjust findings for the different patient characteristics considered. Here we expand findings by examining the entire available cohort and by fitting a multivariable model when considering possible predictors of progression. For the present study, we investigated the natural history of secondary progression in immunomodulatory drug (IMD) naïve patients by examining factors associated with the time from onset to the development of SPMS in the whole BCMS cohort of relapsing at onset patients. Because $\mathrm{MS}$ is a chronic, lifelong disease, the age at which patients reach the progressive phase is important and relevant to patients and their physicians; we have therefore also examined the factors that influence the age at which SPMS develops.

\section{METHODS}

\section{Clinical information}

The clinical information was taken from the British Columbia MS (BCMS) database, a longitudinal database linking the four MS clinics in British Columbia, Canada, and capturing prospective data for over $80 \%$ of the MS population in the province. ${ }^{78}$ We selected all patients with a diagnosis of clinically definite MS according to the Poser diagnostic criteria, ${ }^{9}$ with a relapsing at onset disease course who were enrolled in the database from 1 September 1980 to 31 July 2003. SPMS disease course was defined clinically by the treating neurologist as a progressive course following a relapsing-remitting onset. ${ }^{10}$ Follow-up visits typically occurred annually. In order to investigate the natural history of the disease without the possible influence of IMDs, patients who received such treatments were censored at the first day of treatment. Gender, age at disease onset and onset symptoms (motor, sensory, optic neuritis and cerebellar/ataxia/brainstem), recorded at the patient's first BCMS clinic visit, were also extracted from this database. Age at onset was divided into four categories: $<30$ years, 30 to $<40,40$ to $<50$ and $\geq 50$ years. The main outcome measures were the time from disease onset and from birth to the onset of secondary progression.

\section{Statistics}

The time from disease onset to onset of secondary progression and the age at secondary progression (ie, time from birth to SPMS) were estimated from 
Kaplan-Meier survival analyses. Group differences in survival time were compared with log rank tests. In addition, we built multivariable Cox regression models with time to secondary progression and age at secondary progression as the dependent variables. Covariates included in these models were gender, onset symptoms and age at disease onset for the time from onset to secondary progression, and gender and onset symptoms for the age at secondary progression. The age at disease onset was not included as a covariate in the model for age at secondary progression because its value is necessarily smaller than the outcome in every case. The latter model was stratified by age at disease onset instead. We tested for possible violations of the proportional hazards assumption in these multivariable models with log minus log plots and Schoenfeld residuals.

All statistical analyses were performed with the SPSS statistical software package V.16 (SPSS Inc).

\section{RESULTS}

\section{Patient cohort}

Of 5778 patients with definite MS, 5207 (90\%) had a relapsing-remitting onset. IMDs had been used by 1249 (24\%) patients; these patients were censored at the first day of treatment. The date of onset of secondary progression was unavailable for 38 patients $(0.7 \%)$-these patients were excluded from all analyses. The time of disease onset was unknown for a further seven patients $(0.1 \%)$-these patients were excluded from the analyses of the time to secondary progression from onset of MS and from the stratified Cox regression analysis of predictors of age at SPMS. Of the 5169 available patients, 1821 (35\%) had reached SPMS by the study end.

\section{Kaplan-Meier survival analyses}

The results of the Kaplan-Meier survival analyses are shown in table 1. Median time to secondary progression was 21.4 years from onset ( $95 \%$ CI 20.6 to 22.2 years) and median age at secondary progression was 53.7 years (95\% CI 53.1 to 54.3 years). There was wide variability in survival estimates in both the time to and the age at secondary progression: while the $25 \%$ of patients with the quickest disease progression reached secondary progression in less than 11.4 years and at a younger age than 45.1 years (first quartile estimated from the Kaplan-Meier analysis), the $25 \%$ with the slowest progression reached secondary progression after more than 32 years and at an age of more than 63.1 years (last quartile). Men and patients with motor onset symptoms developed SPMS after a shorter time and at a younger age (see table 1 and figures 1 and 2). Patients with a younger age at onset took a longer time to SPMS but reached SPMS at a younger age $(p<0.0005)$ (figure 3$)$.

\section{Multivariable regression models}

The results of the multivariable Cox regression models are shown in table 1 . Male gender and the presence of motor onset symptoms were independently associated with a shorter time to, and a younger age at, SPMS (table 1). Younger age at disease onset was associated with a longer time to SPMS; the hazard

Table 1 Kaplan-Meier estimates and multivariable HRs of time to and age at secondary progression by potential risk factors

\begin{tabular}{|c|c|c|c|c|c|c|c|c|c|c|}
\hline Factor & $\mathbf{n}$ & $\begin{array}{l}\text { Median time to } \\
\text { SPMS }(95 \% \mathrm{CI})\end{array}$ & p Value & $\begin{array}{l}\text { Time to SPMS } \\
\text { HR† }(95 \% \text { CI) }\end{array}$ & p Value & $\mathbf{n}$ & $\begin{array}{l}\text { Median age at } \\
\text { SPMS (95\% CI) }\end{array}$ & p Value & $\begin{array}{l}\text { Age at SPMS } \\
\mathrm{HR} \dagger(95 \% \mathrm{CI})\end{array}$ & p Value \\
\hline Overall & 5162 & $21.4,20.6$ to 22.2 & - & & & 5169 & $53.7,53.1$ to 54.3 & & & \\
\hline \multicolumn{11}{|l|}{ Gender } \\
\hline Women & 3792 & $22.7,21.8$ to 23.7 & $<0.0005$ & 1.0 (reference) & $<0.0005$ & 3796 & $54.4,53.7$ to 55.2 & $<0.0005$ & 1.0 (reference) & $<0.0005$ \\
\hline Men & 1370 & $17.1,15.4$ to 18.7 & & $1.43,1.30$ to 1.58 & & 1373 & $51.2,49.9$ to 52.5 & & $1.43,1.30$ to 1.58 & \\
\hline Age at onset (years) ${ }^{*}$ & & & & & & $*$ & & & & \\
\hline$<30$ & 2533 & $24.9,23.8$ to 26.0 & $<0.0005$ & $1.05,1.04$ to $1.06 \neq$ & $<0.0005$ & 2533 & $48.6,47.6$ to 49.6 & $<0.0005$ & - & - \\
\hline 30 to $<40$ & 1680 & $18.5,17.3$ to 19.7 & & & & 1680 & $52.8,51.7$ to 53.8 & & & \\
\hline 40 to $<50$ & 761 & $12.2,11.0$ to 13.4 & & & & 761 & $56.5,54.9$ to 58.0 & & & \\
\hline 50 and above & 188 & $8.1,7.0$ to 9.3 & & & & 188 & $64.8,63.0$ to 66.5 & & & \\
\hline \multicolumn{11}{|l|}{ Onset symptoms } \\
\hline \multicolumn{11}{|l|}{ Motor } \\
\hline Absent & 4131 & $22.0,21.1$ to 22.9 & $<0.0005$ & 1.0 (reference) & 0.02 & 4137 & $54.1,53.3$ to 54.9 & 0.31 & 1.0 (reference) & 0.004 \\
\hline Present & 1031 & $18.6,17.1$ to 20.0 & & 1.03 to 1.31 & & 1032 & $52.9,51.7$ to 54.1 & & $1.12,1.06$ to 1.35 & \\
\hline \multicolumn{11}{|l|}{ Sensory } \\
\hline Absent & 2597 & $22.7,21.8$ to 23.5 & 0.22 & 1.0 (reference) & 0.95 & 2603 & $53.4,52.6$ to 54.1 & 0.29 & 1.0 (reference) & 0.68 \\
\hline Present & 2565 & $23.1,21.9$ to 24.2 & & $1.0,0.89$ to 1.13 & & 2566 & $54.7,53.3$ to 55.2 & & $1.03,0.91$ to 1.16 & \\
\hline \multicolumn{11}{|l|}{ CAB } \\
\hline Absent & 4511 & $23.1,22.3$ to 23.9 & 0.60 & 1.0 (reference) & 0.3 & 4518 & $53.9,53.2$ to 54.5 & 0.18 & 1.0 (reference) & 0.15 \\
\hline Present & 651 & $22.0,20.5$ to 23.6 & & $1.08,0.93$ to 1.26 & & 651 & $52.8,50.6$ to 54.9 & & $1.12,0.96$ to 1.30 & \\
\hline \multicolumn{11}{|l|}{ ON } \\
\hline Absent & 4251 & $21.4,20.5$ to 22.3 & 0.71 & 1.0 (reference) & 0.34 & 4258 & $53.9,53.2$ to 54.6 & 0.02 & 1.0 (reference) & 0.15 \\
\hline Present & 911 & $21.4,19.9$ to 23.0 & & $1.08,0.93$ to 1.25 & & 911 & $52.6,51.1$ to 54.2 & & $1.03,0.96$ to 1.30 & \\
\hline
\end{tabular}

*Seven patients for whom date at disease onset was unknown were excluded from all analyses of time to SPMS and from the analysis of the association between age at onset and age at SPMS.

†Derived from Cox regression analyses.

$\ddagger$ HRs represent a per year increase in risk.

$\mathrm{CAB}$, cerebellar, ataxia or brainstem; ON, optic neuritis; SPMS, secondary progressive multiple sclerosis. 


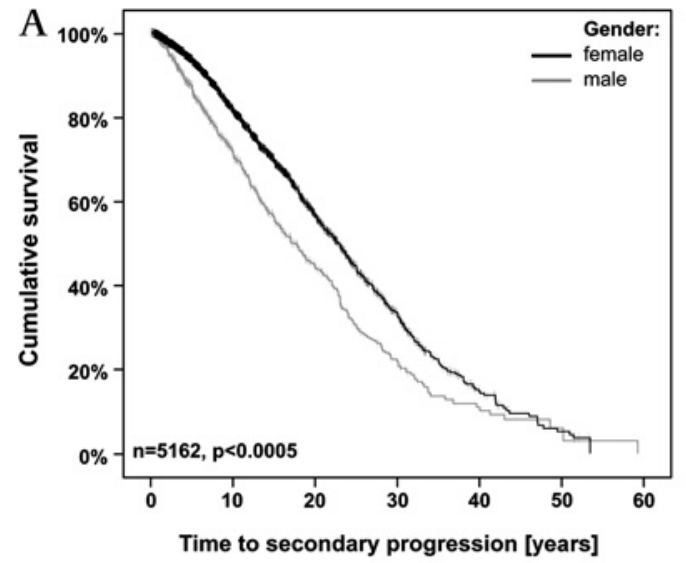

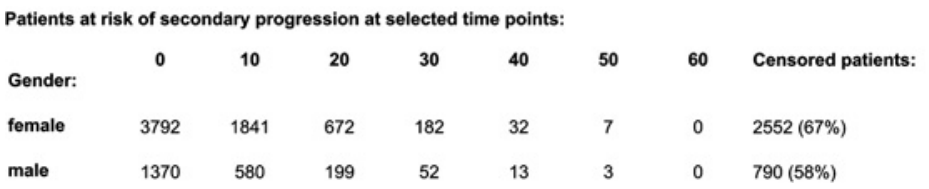

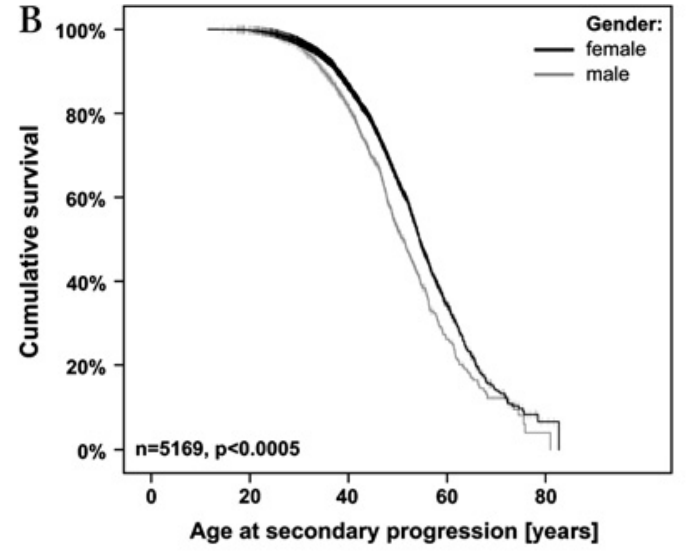

Patients at risk of secondary progression at selected time points:

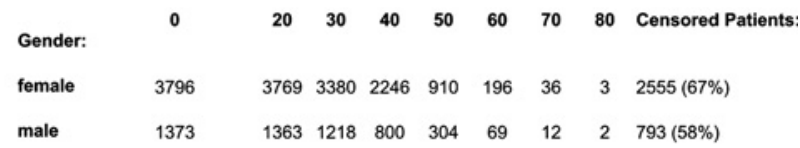

Figure 1 Kaplan-Meier curves showing time to $(A)$ and age at $(B)$ secondary progression by gender.

rate (HR) of reaching SPMS increased by $27.1 \%$ (95\% CI 23.4 to $30.9 \%$ ) for every 5 year increase in onset age (HR per 1 year increase: 1.05 (95\% CI 1.04 to 1.06$)$ ).

Other onset symptoms were not associated with these outcomes. Our investigation of all models with log minus log plots and Schoenfeld residuals showed no evidence of a violation of the proportional hazards assumption

\section{DISCUSSION}

We have reported on the long term follow-up of a cohort of relapsing at onset MS patients from a population based MS database in British Columbia, Canada. We found the onset of secondary progression to be a gradual process in the cohort as a whole, with a steady increase of conversions to SPMS observed over follow-up. However, the window was wide, with SPMS being reached within 1 year of MS onset through to more than 50 years post-onset, and in individuals from 11 to 82 years of age.

Overall, the median time to SPMS was 21.4 years, which is similar to that reported for the few other cohorts in which
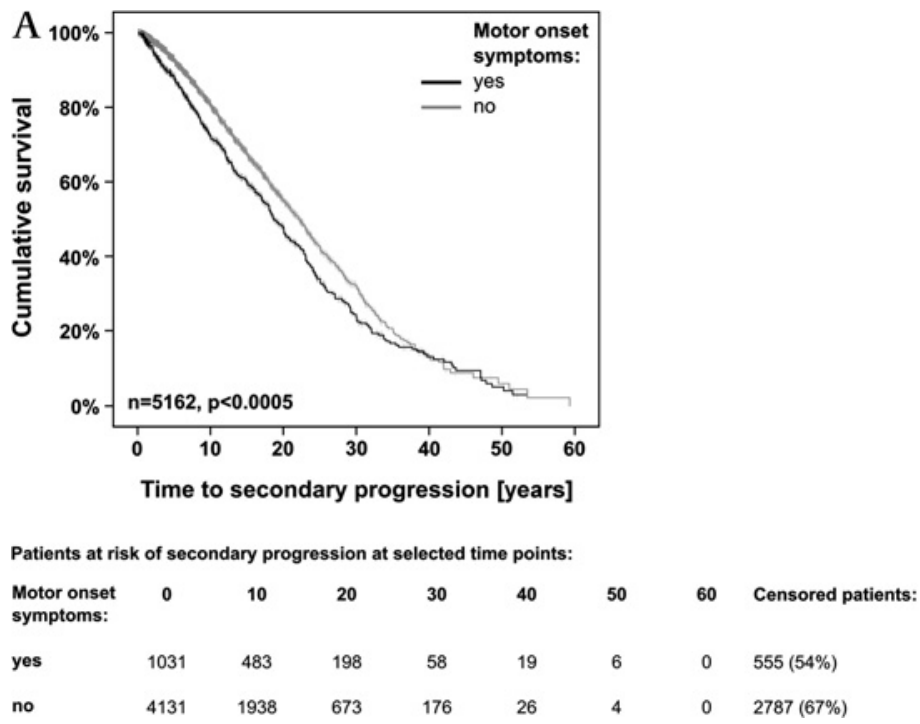

survival analyses have been applied, including 19.1 years in Lyon $^{11}$ and 20.0 years in Lorraine, France. ${ }^{12}$

Three factors were associated with the onset of secondary progression in our cohort: gender, age at onset and motor onset symptoms.

Male gender predicted a shorter time to, and a younger age at, SPMS in our cohort. The influence of male gender on the time to onset of SPMS has been controversial-while some previous studies suggested that men have a worse prognosis than women, ${ }^{6} 1113$ other studies have found no such association. ${ }^{14-18}$ Sex hormones may play a role in this gender difference-an increase in oestrogen levels in particular is believed to reduce CNS inflammation. ${ }^{19}$ Relapse frequency is also reduced during pregnancy and shows a rebound after delivery. This phenomenon has been associated with the rise and fall of oestrogen and progesterone levels during pregnancy and the postpartum period. ${ }^{20}$ Furthermore, the administration of oral oestriol in women with MS resulted in a decrease in the number and volume of gadolinium enhancing lesions on cranial MRI in one
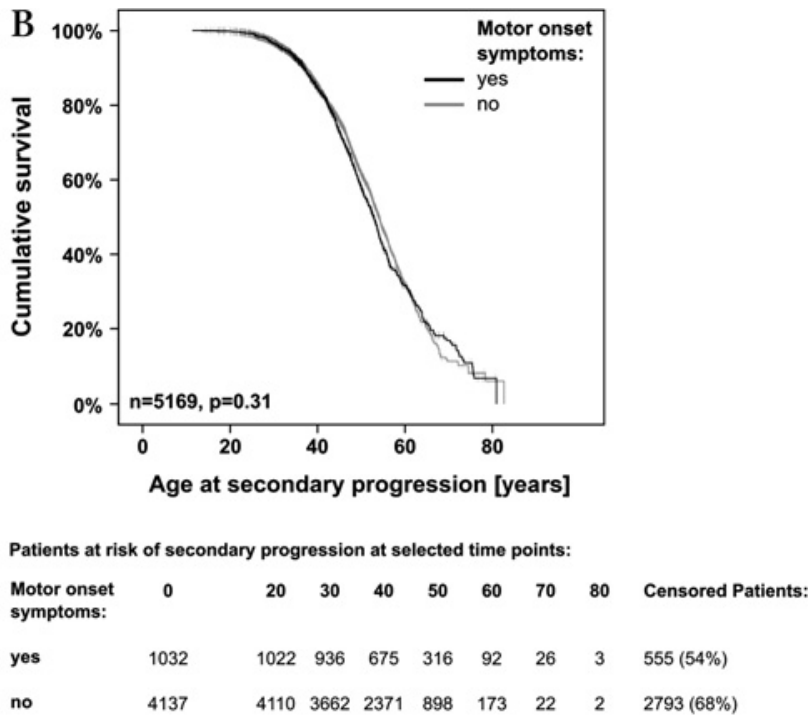

Figure 2 Kaplan-Meier curves showing time to $(A)$ and age at $(B)$ secondary progression by motor onset symptoms. 


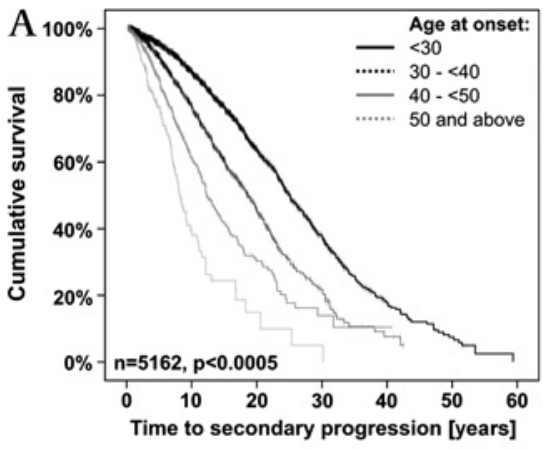

Patients at risk of secondary progression at selected time points:

$\begin{array}{lcccccccc} & \mathbf{0} & \mathbf{1 0} & \mathbf{2 0} & \mathbf{3 0} & \mathbf{4 0} & \mathbf{5 0} & \mathbf{6 0} & \text { Censored patients: } \\ \begin{array}{l}\text { Age at onset: } \\ <30\end{array} & 2533 & 1514 & 659 & 197 & 39 & 10 & 0 & 1635(65 \%) \\ \mathbf{3 0} \text { to }<\mathbf{4 0} & 1680 & 698 & 174 & 31 & 5 & 0 & 0 & 1113(66 \%) \\ \mathbf{4 0} \text { to }<50 & 761 & 184 & 35 & 5 & 1 & 0 & 0 & 490(64 \%) \\ \mathbf{5 0} \text { and above } & 188 & 25 & 3 & 1 & 0 & 0 & 0 & 104(55 \%)\end{array}$

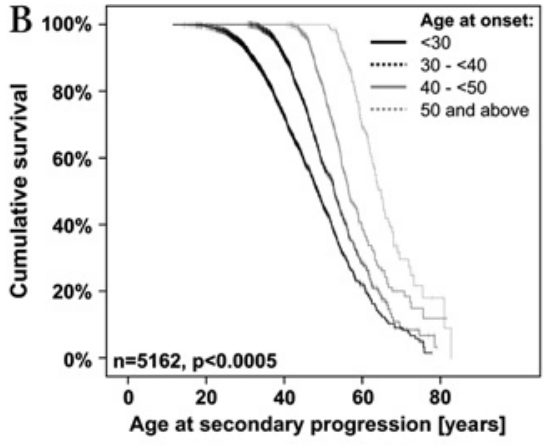

Patients at risk of secondary progression at selected time points:

\begin{tabular}{|c|c|c|c|c|c|c|c|c|c|}
\hline & 0 & 20 & 30 & 40 & 50 & 60 & 70 & 80 & Censored Patients: \\
\hline \multicolumn{10}{|l|}{ Age at onset: } \\
\hline$<30$ & 2533 & 2496 & 1962 & 936 & 299 & 61 & 14 & 0 & $1635(65 \%)$ \\
\hline 30 to $<40$ & - & - & 1680 & 1158 & 341 & 62 & 7 & 0 & $1113(66 \%)$ \\
\hline 40 to $<50$ & - & - & - & 761 & 385 & 64 & 13 & 2 & $490(64 \%)$ \\
\hline 50 and above & - & - & - & - & 188 & 78 & 14 & 3 & $104(55 \%)$ \\
\hline
\end{tabular}

Figure 3 Kaplan-Meier curves showing time to $(A)$ and age at $(B)$ secondary progression by age at disease onset.

preliminary study. ${ }^{21}$ A further trial investigating the potential therapeutic value of sex hormones for MS is currently ongoing. ${ }^{22}$

Motor or 'long tract' onset symptoms have reportedly been associated with the time to SPMS in some studies ${ }^{11} 1416$ while others have found no association between onset symptoms and the timing of secondary progression. ${ }^{13} 1517$ It is not clear why a motor onset should impact the disease course more than other onset symptoms. The steady worsening of neurological signs may be more apparent in patients with a motor disease onset, and this could lead to the earlier detection of secondary progression in this group. It is also possible that a significant portion of those patients with motor onset symptoms have an early involvement of the spinal cord which is believed to carry a worse prognosis. ${ }^{23}$ This merits further investigation.

Age at disease onset is considered to be the strongest predictor of the time to SPMS. ${ }^{11}{ }^{15-17}$ A young age at the onset of MS has long been viewed as a relatively good prognostic sign because a longer delay to specific disability outcomes has typically been observed in these patients. ${ }^{24}$ In terms of the pathophysiology of MS, a younger age has been viewed as favourable because older age is associated with worse recovery from experimental demyelination in animal models due to impaired recruitment and differentiation of oligodendrocyte precursor cells. ${ }^{25}$ Our investigation of the influence of MS onset age on conversion to SPMS shows that a younger age at MS onset should not be viewed as favourable: patients with a young age at disease onset may take longer until they convert to SPMS but they are also younger at the onset of SPMS. This concurs with recent findings from other natural history studies examining time to disability (EDSS) milestones in both adult and paediatric MS. ${ }^{26-28}$

The main strength of this study is the fact that we have investigated a large population based cohort followed for a long time period with prospectively collected data. A limitation of our study, however, is the fact that an estimated $20 \%$ of the MS patients in BC have not been captured by the database; we are unable to determine if these other MS patients in BC would have progressed to SPMS faster or slower than the patients included in this study. On the other hand, there is no reason to believe that factors such as gender, onset symptoms and age at onset would have any different effect on disease course among those not included in the database.
Recent research suggests that the use of IMDs may influence the timing of secondary progression. ${ }^{15} 29$ In this study, we concentrated on untreated patients to evaluate which factors are relevant in the natural history of the disease and to provide a treatment naïve comparison for future cohort studies. This information may also be helpful for the design of randomised controlled trials of the effect of IMDs on conversion to SPMS.

\section{Acknowledgements The statistical advice of Dr Yinshan Zhao is gratefully acknowledged.}

Funding MK received research support from a Canadian Institutes of Health Research (CIHR) Fellowship and a Du Pré Grant from the MS International Federation (MSIF, http://www.msif.org). EK is funded by an MS Society of Canada Post-doctoral Fellowship. PR serves as Research Chair of the MS Society of Canada, serves on scientific advisory boards for the Germany MS Society and receives research support from the MS Society of Canada, the US National MS Society and the CIHR. HT received research support from the CIHR (190898 (PI) and MOP-82738 (PI)), the US National MS Society and the MS Society of Canada (Don Paty Career Development Award), and is a Michael Smith Foundation for Health Research Scholar. The BCMS database was funded by an unrestricted grant from Dr Donald Paty and the MS/MR Research Group.

Competing interests PR serves on scientific advisory boards for Merck Serono, Novartis, Teva Pharmaceutical Industries Ltd and Bayer-Schering Pharma. He also serves on the editorial advisory board of Therapeutics in Neurology and has received speaker and/or consulting honoraria from Bayer-Schering Pharma, Biogen Idec, Merck Serono, Novartis and Teva Pharmaceutical Industries Ltd. HT has received speaker honoraria from the Swiss MS Society and the University of British Columbia MS Research Program.

Ethics approval The study was approved by the clinical research ethics board of the University of British Columbia.

Provenance and peer review Not commissioned; externally peer reviewed.

\section{REFERENCES}

1. Compston A, Coles A. Multiple sclerosis. Lancet 2002;359:1221-31.

2. Rovaris $\mathbf{M}$, Confavreux $\mathbf{C}$, Furlan $\mathrm{R}$, et al. Secondary progressive multiple sclerosis: current knowledge and future challenges. Lancet Neurol 2006;5:343-54.

3. Minderhoud JM, van der Hoeven JH, Prange AJ. Course and prognosis of chronic progressive multiple sclerosis. Results of an epidemiological study. Acta Neurol Scand 1988;78:10-15.

4. Stankoff B, Mrejen S, Tourbah A, et al. Age at onset determines the occurrence of the progressive phase of multiple sclerosis. Neurology 2007;68:779-81.

5. Koch M, Mostert J, Heersema D, et al. Progression in multiple sclerosis: further evidence of an age dependent process. J Neurol Sci 2007;255:35-41.

6. Tremlett H, Yinshan Z, Devonshire V. Natural history of secondary-progressive multiple sclerosis. Mult Scler 2008;14:314-24. 
7. Sweeney VP, Sadovnick AD, Brandejs V. Prevalence of multiple sclerosis in British Columbia. Can J Neurol Sci 1986;13:47-51.

8. Sadovnick AD, Ebers GC, Wilson RW, et al. Life expectancy in patients attending multiple sclerosis clinics. Neurology 1992;42:991-4.

9. Poser CM, Paty DW, Scheinberg L, et al. New diagnostic criteria for multiple sclerosis: guidelines for research protocols. Ann Neurol 1983:13:227-31.

10. Lublin FD, Reingold SC. Defining the clinical course of multiple sclerosis: results of an international survey. National Multiple Sclerosis Society (USA) Advisory Committee on Clinical Trials of New Agents in Multiple Sclerosis. Neurology 1996:46:907-11.

11. Vukusic S, Confavreux C. Prognostic factors for progression of disability in the secondary progressive phase of multiple sclerosis. J Neurol Sci 2003;206:135-7.

12. Debouverie M, Louis S, Pittion-Vouyovitch S, et al. Multiple sclerosis with a progressive course from onset in Lorraine-Eastern France. J Neurol 2007;254:1370-5.

13. Bergamaschi R, Berzuini $\mathrm{C}$, Romani $\mathrm{A}$, et al. Predicting secondary progression in relapsing-remitting multiple sclerosis: a Bayesian analysis. J Neurol Sci 2001:189:13-21.

14. Amato MP, Ponziani G, Bartolozzi ML, et al. A prospective study on the natural history of multiple sclerosis: clues to the conduct and interpretation of clinical trials. J Neurol Sci 1999;168:96-106.

15. Koch M, Uyttenboogaart M, van Harten A, et al. Factors associated with the risk of secondary progression in multiple sclerosis. Mult Scler 2008;14:799-803.

16. Trojano M, Avolio C, Manzari C, et al. Multivariate analysis of predictive factors of multiple sclerosis course with a validated method to assess clinical events. J Neurol Neurosurg Psychiatry 1995;58:300-6.

17. Riise T, Gronning M, Fernandez 0 , et al. Early prognostic factors for disability in multiple sclerosis, a European multicenter study. Acta Neurol Scand 1992:85:212-18.
18. Runmarker B, Andersen 0. Prognostic factors in a multiple sclerosis incidence cohort with twenty-five years of follow-up. Brain 1993;116:117-34.

19. Schwendimann RN, Alekseeva N. Gender issues in multiple sclerosis. Int Rev Neurobiol 2007:79:377-92.

20. Confavreux C, Hutchinson M, Hours MM, et al. Rate of pregnancy-related relapse in multiple sclerosis. Pregnancy in Multiple Sclerosis Group. N Engl J Med 1998;339:285-91.

21. Sicotte NL, Liva SM, Klutch $\mathrm{R}$, et al. Treatment of multiple sclerosis with the pregnancy hormone estriol. Ann Neurol 2002:52:421-8.

22. Vukusic S, lonescu I, El Etr M, et al. The Prevention of Post-Partum Relapses with Progestin and Estradiol in Multiple Sclerosis (POPART'MUS) trial: rationale, objectives and state of advancement. J Neurol Sci 2009;286:114-18.

23. Kidd D, Thorpe JW, Thompson AJ, et al. Spinal cord MRI using multi-array coils and fast spin echo. II. Findings in multiple sclerosis. Neurology 1993:43:2632-7.

24. Compston A, Ebers GC, Lassmann H, et al. McAlpine's multiple sclerosis. 3rd Edn. London: Churchill Livingstone, 1998.

25. Sim FJ, Zhao C, Penderis J, et al. The age-related decrease in CNS remyelination efficiency is attributable to an impairment of both oligodendrocyte progenitor recruitment and differentiation. J Neurosci 2002;22:2451-9.

26. Confavreux C, Vukusic S. Age at disability milestones in multiple sclerosis. Brain 2006:129:595-605

27. Renoux C, Vukusic S, Mikaeloff $Y$, et al. Natural history of multiple sclerosis with childhood onset. N Engl J Med 2007;356:2603-13.

28. Tremlett H, Paty D, Devonshire V. Disability progression in multiple sclerosis is slower than previously reported. Neurology 2006;66:172-7.

29. Trojano M, Pellegrini F, Fuiani A, et al. New natural history of interferon-beta-treated relapsing multiple sclerosis. Ann Neurol 2007:61:300-6. 